\title{
Evaluación de arcillas caoliniticas-illiticas provenientes de la formación guayabo del Área Metropolitana de Cúcuta, Norte de Santander, Colombia
}

\section{Evaluation kaolinitic-illiticas clays from the guayabo formation of the Cucuta's Metropolitan Area, Norte de Santander (Colombia)}

\author{
Avaliação de argilas cauliníticas - illíticas da origem \\ "formação guayabo" da Área metropolitana de \\ Cúcuta, Norte de Santander, Colômbia
}

\author{
Vivianne Isel Cáceres ${ }^{1}$; Jorge Sánchez-Molina ${ }^{2 *}$; Amanda Lucía Chaparro-García ${ }^{3}$ \\ ${ }^{1}$ Maestría en Química, Facultad de Ciencias Básicas, ${ }^{3}$ Grupo de Investigación de Recursos Naturales, Universidad \\ de Pamplona, Pamplona, Colombia. \\ ${ }^{2}$ Docente titular departamento de química. Centro de Investigación de Materiales Cerámicos CIMAC, Grupo de \\ Investigación en Tecnología Cerámica GITEC, Universidad Francisco de Paula Santander, Cúcuta, Colombia. \\ * jorgesm@ufps.edu.co
}

Fecha Recepción: 06 de octubre de 2016 Fecha Aceptación: 19 de julio de 2017

\begin{abstract}
Resumen
La caracterización de arcillas procedentes de la Formación Guayabo del Área Metropolitana de Cúcuta, Norte de Santander (Colombia) es presentada. Los resultados obtenidos de estos análisis permiten determinar las propiedades de estas arcillas y potenciar su uso en la fabricación de materiales de construcción y en la elaboración de nuevos materiales cerámicos, generando impactos tecnológicos, económicos y sociales en la región, pues se podrá competir con productos de calidad en los exigentes mercados nacionales e internacionales. Es así que a través de este trabajo se realizó la caracterización química, mineralógica, térmica y físicocerámica, para determinar la estructura, composición y comportamiento de estas materias primas. Las muestras se obtuvieron de dos puntos diferentes de la formación, ubicados en los municipios del Zuliay Villa del Rosario. Para la evaluación se utilizó la información obtenida de la caracterización química mediante Fluorescencia de Rayos X (FRX), caracterización mineralógica mediante Difracción de Rayos X (DRX) e infrarrojo con transformada de Fourier (FTIR), caracterización térmica mediante análisis térmico gravimétrico (TGA) y calorimetría diferencial de barrido (DSC); y finalmente caracterización físico-cerámica. Los resultados indican que los minerales arcillosos están constituidos por aluminosilicatos hidratados con presencia de algunas impurezas como sodio, hierro, potasio, calcio, titanio por lo que presentan capacidad de ser sometidas a esfuerzos para su conformado a través de procesos de extrusión. Se evidencia que aunque ambas muestras se encuentran en diferentes municipios del área metropolitana de Cúcuta, presentan características similares en fases cristalinas, grupos funcionales, comportamientos térmicos y propiedades cerámicas.
\end{abstract}

Palabras clave: material arcilloso, DRX, FRX, FTIR, TGA

\section{Abstract}

The characterization of clays from the Guayabo Formation at the Cúcuta Metropolitan Area, Norte de Santander (Colombia) is presented. The results of these analyzes allow to determine the properties of these clays and to enhance their use in the manufacture of building materials and in the development of new ceramic materials, creating technological, economic and social impacts in the region, as they can 
compete with high quality product in the demanding national and international markets. Thus, through this work, chemical, mineralogical, thermal and physical-ceramic characterizations were performed to determine the structure, composition and behavior of these raw materials. The samples were obtained from two different points of the geological formation, located at the municipalities of Zulia and Villa del Rosario. The information obtained from the chemical characterization, mineralogical, thermal and physicalceramic, using the techniques of X-ray fluorescence, X-ray diffraction, thermal gravimetric analysis, Differential Scanning Calorimetry and Infrared Fourier Transform, was used for evaluation. The results indicate that the clay minerals are composed of hydrous aliminosilicates with presence of some impurities such as sodium, iron, potasium, calcium, titanium and this have ability to be subjected to efforts for shaping by extrusion processes. It is shown that although both samples are from different municipalities in the metropolitan area of Cucuta, have similar characteristics in crystalline phases, functional groups, thermal behavior and ceramic properties.

Keywords: clayey material, XRD, XRF, FTIR, TGAA

\section{Resumo}

A caracterização das argilas da formação Guayabo da Área Metropolitana, Cúcuta Norte de Santander (Colômbia) é apresentado. Os resultados destas análises permitem a determinação das propriedades destas argilas e promovem a sua utilização na fabricação de materiais de construção e no desenvolvimento de novos materiais cerâmicos, gerando impactos tecnológicos, económicos e sociais na região, Assim, estes poderão competir com os produtos de qualidade em os exigentes mercados nacionais e internacionais. Então, com o desenvolvimento deste trabalho, foi possível a caracterização química, mineralógica, térmica e físico-cerâmica para determinar a estrutura, composição e comportamento destes materiais. As amostras foram obtidas de dois pontos diferentes da formação, localizadas no município "El Zulia" e "Villa del Rosario". Para a avaliação se utilizou a informação obtida da caracterização química através de Fluorescência de Raios-X (FRX), a caracterização mineralógica através de Difração de Raios-X (DRX) e infravermelho com transformada de Fourier (FTIR), a caracterização térmica foi feita através de análise térmico gravimétrico (TGA) e calorimetria de varredura diferencial (DSC), e finalmente a caracterização físico-cerâmica. Os resultados indicarem que os minerais argilosos consistem de alumino-silicato hidratado com a evidência de algumas impurezas como sódio, ferro, potássio, cálcio e titânio; é assim então, que estes materiais apresentam capacidade de ser submetidos a esforços para seu formado através de processos de extrusão. No entanto, é evidente que embora ambas amostras se coletaram em diferentes municípios da área metropolitana de Cúcuta apresentaram características semelhantes em fases cristalinas, grupos funcionais, nos seus comportamentos térmicos e nas suas propriedades cerâmicas.

Palavras-chave: DSC, FTIR, material argiloso, TGA, XRD, XFR

\section{Introducción}

Las arcillas son minerales naturales que están compuestas de múltiples silicoaluminatos hidratados que contienen iones tales como K, Fe, $\mathrm{Mg}$ y $\mathrm{Na}$, y otros minerales como feldespatos, cuarzo, anatasa, rutilo, hematita, carbonatos, entre otros; se encuentran en la naturaleza generalmente acompañadas por materia orgánica [1,2]. En Colombia las arcillas se usan principalmente para la fabricación de materiales de construcción y alfarería [3].

Cúcuta y su Área Metropolitana se encuentra privilegiada por poseer formaciones arcillosas de excelente calidad [4], lo que ha permitido dar origen a uno de los sectores más significativos de la región: la industria cerámica, conformado por 49 empresas legalmente establecidas, todas ellas dedicadas a la fabricación de productos a base de arcilla, de los que se destacan los materiales de la construcción, tales como: cerámica, tableta vitrificada, tableta esmaltada, tejas, ladrillos, productos artesanales (decorados y enchapes), entre otros [5]; convirtiéndose en una de las principales alternativas de desarrollo para la región, es por ello que es de gran importancia mejorar constantemente la calidad de los productos ofertados con el fin de alcanzar la competitividad necesaria para mantenerse en los mercados actuales e incursionar en otros a nivel internacional [6]. 
Es importante destacar que, hasta el momento, en la literatura se encuentran pocos estudios químicos, térmicos y/o mineralógicos completos reportados para las arcillas de Norte de Santander [6]. Por lo tanto, el sector cerámico de Cúcuta y su Área Metropolitana no tienen caracterizadas sus arcillas química, térmica y mineralógicamente, sólo se cuentan con estudios de caracterización físico cerámico y tecnológico de algunas arcillas de la región; además sus procesos de producción son poco tecnificados. Norte de Santander posee depósitos de minerales arcillosos muy extensos aproximadamente de $2196 \mathrm{~km}^{2}$ que corresponde al $10,1 \%$ de la extensión departamental y las principales arcillas explotadas en el departamento se encuentran en el Área Metropolitana de Cúcuta [7].

La agenda interna para la productividad y la competitividad de Norte de Santander propone que para el 2020, el sector de la cerámica deberá ser el líder nacional y binacional en el campo de los productos cerámicos de alta calidad [8]. Para esto, se quieren aprovechar ventajas, tales como: proximidad con la frontera, alta concentración geográfica de las industrias cerámicas, mano de obra económica y cualificada, competitividad de los precios frente a otras regiones y la calidad de las arcillas de la región. Para cumplir con ésta meta, la tecnificación del sector cerámico en la región es imprescindible y ésta se logra únicamente mediante el conocimiento de las propiedades tanto físicas como químicas de las arcillas, y de los productos terminados.

Los resultados obtenidos de la investigación permiten conocer las propiedades físicocerámicas, tecnológicas, el comportamiento térmico y la composición química y mineralógica, lo cual sirve de base para futuras investigaciones con el fin de optimizar los procesos de fabricación de productos cerámicos para la construcción y de esta manera darle valor agregado, y a su vez permite evaluar su aptitud de uso en los diferentes campos de utilización de este material tan valioso propiciando la explotación correcta del mismo.

\section{Materiales y métodos}

Las muestras se obtuvieron de dos puntos diferentes de la Formación Guayabo del Área Metropolitana de Cúcuta, uno ubicado en el municipio del Zulia y el otro en el municipio de Villa del Rosario, la distancia entre los dos puntos de la toma de la muestra es de $24 \mathrm{~km}$.
Para confirmar la formación geológica de la cual se estaba obteniendo la muestra, se tomaron las coordenadas con un GPS y posteriormente se localizaron en el mapa geológico del Cuadrángulo G-13 de Cúcuta, el cual representa la estratigrafía de Cúcuta y sus alrededores.

Las muestras se prepararon por vía seca, fue necesario realizar un secado previo a una temperatura que no superó los $110^{\circ} \mathrm{C}$, para esto se introdujo la muestra en una estufa de secado hasta lograr disminuir la humedad hasta obtener peso constante. Posteriormente las muestras se trituraron de forma manual hasta obtener un tamaño de partícula menor a $2 \mathrm{~cm}$, y luego se molieron en un molino de martillos para reducir aún más el tamaño de partícula. Finalmente se tamizaron utilizando un tamiz malla ASTM 10 (apertura de $2 \mathrm{~mm}$ ), con el fin de obtener la muestra con un tamaño de partícula menor a 2 $\mathrm{mm}$, estas muestras se conservaron para realizar los respectivos análisis.

El material arcilloso obtenido se caracterizó utilizando FRX, con el fin de obtener un análisis cuantitativo de los componentes químicos que conforman las muestras de arcillas. Para este estudio, las muestras se calcinaron con el fin de que todos los elementos se llevaran a su máximo estado de oxidación formando los respectivos óxidos; los análisis cuantitativos se realizaron mediante el método QUANT-EXPRESS (parámetros fundamentales) en el rango de sodio $(\mathrm{Na})$ a uranio $(\mathrm{U})$, en un espectrómetro secuencial de Fluorescencia de rayos $X$ de longitud de onda dispersiva de 4kW marca BRUKER modelo S8 TIGER. Para detectar los elementos pesados se utilizó un detector de Centelleo y para los elementos livianos un detector de flujo, la fuente de rayos $X$ fue un tubo de Rodio (Rh) y el goniómetro utilizado fue de alta precisión para ángulos theta y 2 theta. La identificación y cuantificación de las fases cristalinas arcillosas y no arcillosas se realizaron mediante la técnica de difracción de rayos $X$ (DRX). Los difractogramas de las muestras fueron obtenidos en un difractómetro de polvo marca BRUKER modelo D8 ADVANCE con geometría DaVinci, utilizando radiación de CuKa1 en el rango $3,5-70^{\circ} 2 \theta$. El análisis cualitativo de las fases presentes en la muestra se realizó mediante comparación del perfil observado con los perfiles de difracción reportados en la base de datos PDF-2 del International Centre for Diffraction Data (ICDD). El análisis cuantitativo de las fases encontradas en la muestra se realizó mediante el 
refinamiento por el Método de Rietveld del perfil observado habiéndole agregado a la muestra el $20 \%$ del estándar interno (óxido de aluminio).

Los principales grupos funcionales de las muestras se determinaron mediante espectroscopía infrarroja (FTIR); para esto, las muestras se maceraron con bromuro de potasio 1:100 y luego se prensaron en forma de pastillas. La medición se llevó a cabo en un espectrofotómetro SHIMADZU IR PRESTIGE-2.1. Los espectros se obtuvieron en la región del infrarrojo medio, con un barrido de $4000 \mathrm{~cm}^{-1}$ hasta $400 \mathrm{~cm}^{-1}$ con una resolución de $4 \mathrm{~cm}^{-1}$. El análisis cualitativo de los grupos funcionales presentes en la muestra se realizó por comparación directa con los espectros de infrarrojo reportados en la literatura.

Los cambios químicos y físicos durante el calentamiento de las muestras se identificaron mediante las técnicas de calorimetría diferencial de barrido (DSC) y análisis termogravimétrico (TGA). Las mediciones de TG y DSC se realizaron en un equipo de análisis térmico simultáneo marca TA Instruments SDT Q600, la muestra pulverizada se colocó en una celda de alúmina, en una atmósfera de aire sintético cuyo caudal fue de $100 \mathrm{~mL} / \mathrm{min}$ y la velocidad de calentamiento fue de $10^{\circ} \mathrm{C} / \mathrm{min}$ hasta alcanzar $1200^{\circ} \mathrm{C}$; se trabajó hasta esta temperatura con el fin de asemejar los procesos a nivel industrial. El objetivo de realizar estos análisis fue determinar el flujo de calor (DSC) y los cambios de peso (TGA) que presenta la muestra como función de la temperatura o el tiempo en una atmósfera controlada.

Para la caracterización físico-cerámica, se utilizó como método de conformado la extrusión, ya que la mayoría de empresas que hay en la región utilizan este método. El agua de amasado se calculó en función de la plasticidad de la muestra, se utilizó una boquilla rectangular y se hicieron probetas de $10 \mathrm{~cm}$ de longitud.

El análisis de retenido sobre tamiz se realizó teniendo en cuenta el procedimiento establecido en la norma ASTM C325 y en el manual para el control de la calidad de materias primas arcillosas [9], el análisis granulométrico por hidrómetro se realizó siguiendo la metodología propuesta en la norma ASTM D422 y el índice de plasticidad se realizó mediante el Método de Pfefferkorn teniendo en cuenta el procedimiento mencionado en el manual para el control de la calidad de materias primas arcillosa [9]. Así mismo, se midió la contracción lineal en secado y cocido; la contracción en secado se hizo con probetas de dimensiones conocidas las cuales se sometieron a secado a $105^{\circ} \mathrm{C}$ durante 24 horas y la contracción en cocido se realizó teniendo como dimensiones iniciales de las probetas secas y las finales de las probetas cocidas. El cálculo de la contracción se hizo midiendo la diferencia de dimensiones y determinando así el porcentaje de contracción de las probetas [9], y con los datos de diferencia de masa en seco y en cocido se determinó las pérdidas por calcinación. La absorción de agua se determinó siguiendo la metodología planteada en la norma NTC 4321-3 [10].

\section{Resultados y Discusión}

\section{Fluorescencia de Rayos $\mathbf{X}$}

El análisis químico de las muestras se realizó utilizando fluorescencia de rayos $\mathrm{X}$, los resultados de la composición química de las muestras se evidencian en la Tabla 1.

Tabla 1. Resultados de FRX obtenidos para las muestras de interés.

\begin{tabular}{ccc}
\hline Elemento & Villa del Rosario (\%) & Zulia (\%) \\
\hline $\mathbf{S i}$ & 28,03 & 28,96 \\
$\mathbf{A l}$ & 11,86 & 10,62 \\
$\mathbf{F e}$ & 3,46 & 4,73 \\
$\mathbf{K}$ & 1,30 & 1,29 \\
$\mathbf{T i}$ & 0,53 & 0,53 \\
$\mathbf{M g}$ & 0,44 & 0,47 \\
$\mathbf{~ N a}$ & 0,27 & 0,13 \\
$\mathbf{C a}$ & 0,21 & 0,09 \\
$\mathbf{P}$ & 0,07 & 0,06 \\
$\mathbf{B a}$ & 0,04 & 0,04 \\
$\mathbf{V}$ & 0,02 & 0,03 \\
$\mathbf{Z r}$ & 0,02 & 0,02 \\
\hline
\end{tabular}

De la Tabla 1 se destacan los elevados contenidos de silicio y aluminio, al igual que el alto porcentaje de hierro lo que justifica el color rojo característico de las arcillas del Área Metropolitana de Cúcuta. Teniendo en cuenta los resultados que se muestran en la Tabla 1 y Tabla 2, y realizando un análisis estequiométrico de las fases cristalinas, se puede deducir que en la muestra de Villa del Rosario el $27,29 \%$ del silicio determinado es cristalino y el $0,75 \%$ es amorfo, y para la muestra del Zulia el $25,60 \%$ es cristalino y el $3,36 \%$ es amorfo; así mismo la relación cristalino - amorfo de la cantidad de aluminio en la muestra de Villa del Rosario y Zulia es, $8,05 \%$ - 3,81\% y $8,50 \%$ - 3,81\%, respectivamente. Por ende, la muestra contiene cantidades considerables de silicoaluminatos y cuarzo $[1,11]$. 
Los valores correspondientes a pérdidas por ignición fueron del 7,31 y $8,44 \%$, para la muestra de Villa del Rosario y El Zulia, respectivamente; estos valores reflejan que las muestras tienen un bajo contenido de material orgánico, y esto es favorable, ya que a menor cantidad de materia orgánica se evita que en el proceso de la cocción queden espacios vacíos por el consumo de este material [12].

\section{Difracción de Rayos X}

Los difractogramas de Rayos $X$ obtenidos para las muestras Villa del Rosario y El Zulia se presentan en la Figura 1. Con base en estos se pueden identificar las fases cristalinas mayoritarias y minoritarias que componen cada una de las muestras.

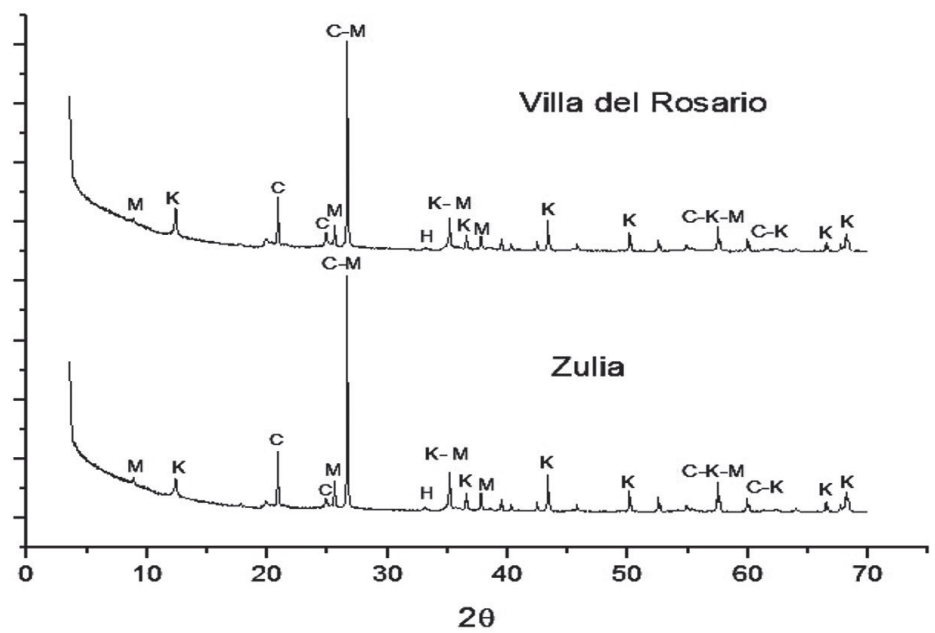

Figura 1. Difractograma de Rayos $X$ de las muestras Villa del Rosario y El Zulia. Caolinita (K); Moscovita (M); Cuarzo (C); Hematita (H).

En la Figura 1 se muestran los difractogramas de Rayos X correspondientes a cada una de las muestras analizadas, se puede observar que son muy similares entre sí, algunos presentan pequeñas variaciones de intensidad; pero considerando la ubicación de los picos se determina la presencia principalmente del cuarzo, caolín, moscovita, microclina y hematita. Los resultados indican que los minerales arcillosos están constituidos por aluminosilicatos hidratados con presencia de algunas impurezas como sodio, hierro, potasio, calcio, titanio, entre otros, los cuales se ratifican con los resultados obtenidos por FRX.

Las fases cristalinas que componen las muestras están reflejadas en la Tabla 2.

Tabla 2. Resultados de DRX obtenidos para las muestras de interés.

\begin{tabular}{ccc}
\hline Fase & Zulia (\%) & Villa del Rosario (\%) \\
\hline Cuarzo & 36,1 & 40,6 \\
Caolinita & 11,7 & 22,8 \\
Hematita & 2,0 & 1,3 \\
Anatasa & 0,3 & $\mathrm{NC}$ \\
Moscovita & 31,0 & 16,8 \\
Microclina & $\mathrm{NC}$ & $\mathrm{NC}$ \\
Total Cristalino & 81,1 & 81,5 \\
Amorfos y otros & 18,9 & 18,5 \\
\hline
\end{tabular}


Las arcillas analizadas tienen carácter caoliniticaillitica, la plasticidad de estos minerales es menor que el de las esmectitas, pero su plasticidad aumenta a medida que disminuye el tamaño del grano; la presencia de potasio en la estructura de la illita (moscovita) le genera cierto poder fundente en el proceso cerámico. La presencia de cuarzo le otorga a la pieza cerámica un control dimensional [13].

\section{Infrarrojo con transformada de Fourier}

En la Figura 2 se muestran los espectros FTIR correspondientes a las muestras de Villa del Rosario y El Zulia sin tratamiento térmico.

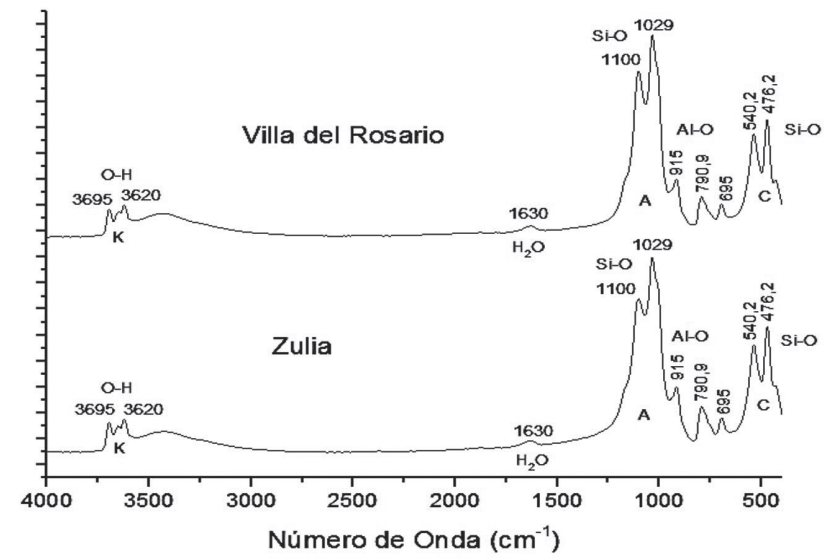

Figura 2. Espectros IR de las muestras arcillosas estudiadas. Caolinita (K); Aluminosilicatos (A); Cuarzo (C); Agua $\left(\mathrm{H}_{2} \mathrm{O}\right)$.

En el estudio de IR de las arcillas analizadas, las vibraciones de estiramiento del enlace $\mathrm{Si}-\mathrm{O}$ fueron observadas a $790,8 \mathrm{~cm}^{-1}, 692,1 \mathrm{~cm}^{-1}, 538,1 \mathrm{~cm}^{-1}$ y $468,7 \mathrm{~cm}^{-1}$ mostrando la presencia de cuarzo [14]. Las bandas tales como $1031,9 \mathrm{~cm}^{-1}, 914,2 \mathrm{~cm}^{-1}, 790,8 \mathrm{~cm}^{-1}$, $692,1 \mathrm{~cm}^{-1}, 538,1 \mathrm{~cm}^{-1}, 468,7 \mathrm{~cm}^{-1}$ muestran la presencia de caolinita [15,16]; las vibraciones observadas a $914,2 \mathrm{~cm}^{-1}$ indican la presencia de hematita. Las bandas a $1031,9 \mathrm{~cm}^{-1}, 914,2 \mathrm{~cm}^{-1}$ (vibraciones de flexión del enlace Al-Al-OH), $790,8 \mathrm{~cm}^{-1}$ y $468,7 \mathrm{~cm}^{1}$ (vibración de flexión del enlace O-Si-O) indican la presencia de illita [17]. La banda grande cerca de $1035 \mathrm{~cm}^{-1}$ corresponde a la vibración $\mathrm{Si}-\mathrm{O}$ estiramiento, las bandas de flexión están en $540-555 \mathrm{~cm}^{-1}$ para el $\mathrm{Si}-\mathrm{O}-\mathrm{Al}$ y en 425 a $433 \mathrm{~cm}^{-1}$ para el Si-O-Si que son características de los silicoaluminatos [11].

En el estudio de los espectros FTIR para las muestras se identificaron varias formas de minerales presentes en la arcilla; se tuvo en cuenta las bandas observadas en el rango de 400 a $1400 \mathrm{~cm}^{-1}$ y han sido asignadas tal como se muestra en la Tabla 3.

Tabla 3. Bandas IR importantes de las muestras de arcillas analizadas con sus asignaciones.

\begin{tabular}{cc}
\hline Banda $\mathbf{~ c m}^{-1}$ & Asignaciones \\
\hline 468,7 & Si-O Estiramiento \\
& Si-O-Fe Estiramiento \\
& O-Si-O Flexión \\
538,1 & Si-O Estiramiento \\
& Si-O-Al Estiramiento \\
692,1 & Si-O Estiramiento \\
& Si-O-Al Estiramiento \\
& Si-O Estiramiento \\
790,8 & Si-O-Al Estiramiento \\
& (Al, Mg)---O-H \\
& Si-O-(Mg, Al) Estiramiento \\
914,2 & Al---O-H Estiramiento \\
& Al-Al-OH Flexión \\
& Si-O-Si \\
& Si-O Estiramiento \\
\hline
\end{tabular}


Es importante destacar que los resultados obtenidos por DRX se relacionaron con los resultados de FTIR, ya que a través de la técnica de IR se confirmaron las fases cristalinas que presentan las muestras.

\section{Caracterización térmica}

La importancia de los análisis térmicos radica, en que permite conocer el comportamiento de la muestra frente a los cambios de temperatura, por lo tanto, a través del perfil térmico se tiene conocimiento para implementar una curva de cocción o cronograma de calentamiento que sea adecuada para la muestra. Al controlar la curva de cocción se puede garantizar que los procesos de sinterización se lleven de manera controlada y se alcance la mayor densificación de las piezas, logrando obtener productos que cumplan con los estándares de calidad que exige el mercado.

Para determinar el efecto del tratamiento térmico en las muestras, se realizaron ensayos de TGA y DSC. En las curvas TGA, DTG y DSC de las arcillas estudiadas mostradas en las Figura 3, se observan los siguientes efectos: el primer pico endotérmico a $125^{\circ} \mathrm{C}-140^{\circ} \mathrm{C}$, lo cual demuestra la eliminación de agua débilmente unida (deshidratación). El segundo pico exotérmico aproximadamente a $300^{\circ} \mathrm{C}$, correspondiente a la descomposición de la materia orgánica y la tercera reacción endotérmica debido a la deshidroxilación en $545^{\circ} \mathrm{C}[2,11]$. Según la literatura, en Colombia en el municipio de Guapi del departamento del Cauca, Muñoz y colaboradores realizaron algunos estudios de caracterización de arcillas en esa región, pero en su artículo no definen la formación geológica a que pertenecen las mismas y trabajaron hasta $800^{\circ} \mathrm{C}$; la muestra de GUAPI I presentó cuatro pérdidas de peso $\left(\sim 77^{\circ} \mathrm{C}\right.$, $\sim 231^{\circ} \mathrm{C}, \sim 324^{\circ} \mathrm{C}, \sim 500^{\circ} \mathrm{C}$ ) con un total de pérdida de peso del $10,52 \%$ y GUAPI II tres pérdidas de peso $\left(\sim 94^{\circ} \mathrm{C}, \sim 265^{\circ} \mathrm{C}, \sim 510^{\circ} \mathrm{C}\right)$ con un total de pérdida de peso del $10,22 \%$. En los resultados de las muestras analizadas en este trabajo, se encontraron tres importantes pérdidas $\left(\sim 125^{\circ} \mathrm{C}\right.$, $\sim 300^{\circ} \mathrm{C}, \sim 545^{\circ} \mathrm{C}$ ), las cuales se encuentran en las mismas regiones de temperaturas que las de Guapi; pero es importante resaltar que las arcillas analizadas del área metropolitana de Cúcuta, no coinciden con los porcentajes de pérdida de peso total, las muestras del Zulia presentaron un 3,87\% y las de Villa del Rosario del $5,6 \%$ hasta $800^{\circ} \mathrm{C}$. Esto nos indica que las arcillas de esta región de Norte de Santander, de la formación Guayabo, son de mejor calidad, pues tendrán menor contracción y menos fisuración, favoreciendo la calidad y estabilidad del producto terminado $[2,18]$.

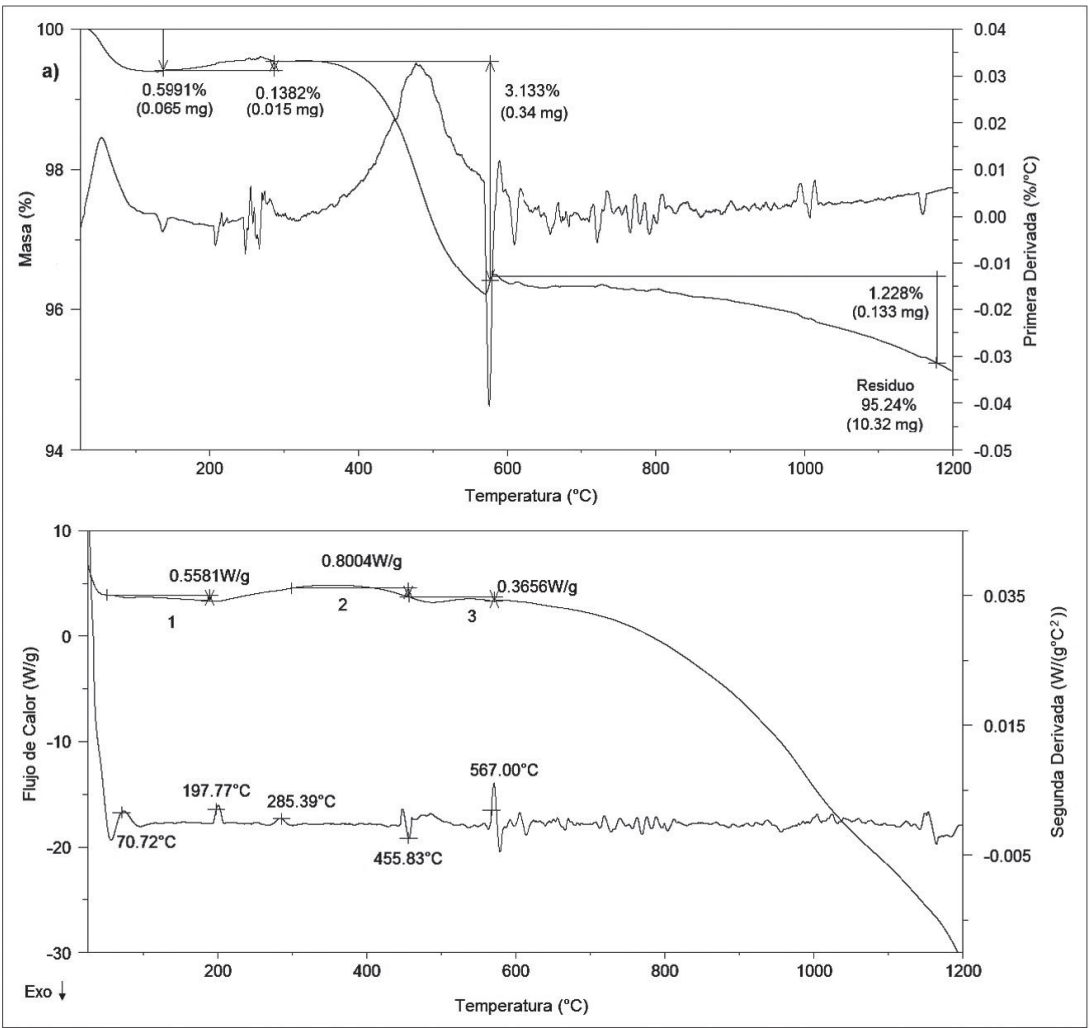

Figura 3. a) Curva TGA, DTG y DSC de la muestra arcillosa El Zulia. 


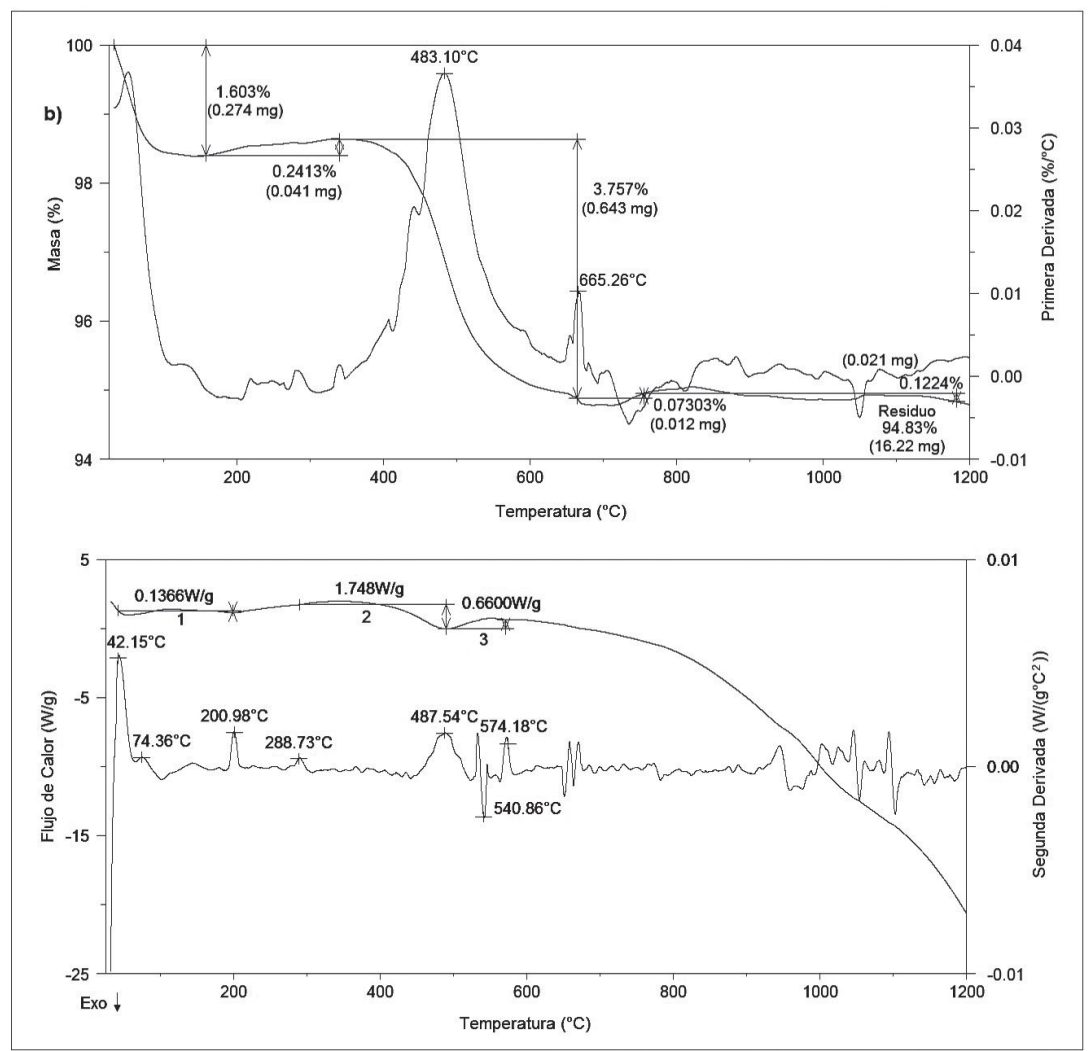

Figura 3. b) Curva TGA, DTG y DSC de la muestra arcillosa Villa del Rosario.

\section{Caracterización físico-cerámica}

Caracterizar las materias primas en la industria cerámica es muy importante ya que permite desarrollar los estándares de calidad que deben cumplir el producto terminado, al conocer las propiedades del material arcilloso es posible realizar el control del proceso productivo [19].

En la Tabla 4 se muestran los resultados obtenidos de la caracterización físico-cerámica.

Tabla 4. Caracterización físico-cerámica.

\begin{tabular}{ccc}
\hline Propiedad & Villa Rosario (\%) & Zulia (\%) \\
\hline Retenido sobre tamiz ASTM 230 & 13,88 & 13,52 \\
$\%$ de arenas & 29,11 & 29,55 \\
$\%$ de limos & 32,24 & 32,71 \\
$\%$ de arcilla & 38,65 & 37,74 \\
Índice de plasticidad & 24,50 & 24,50 \\
Absorción de agua $1150^{\circ} \mathrm{C}$ & 7,51 & 7,94 \\
Contracción en cocido a $1150^{\circ} \mathrm{C}$ & 2,88 & 2,71 \\
Contracción en secado a $1150^{\circ} \mathrm{C}$ & 10,39 & 11,38 \\
Pérdidas por Calcinación a $1100^{\circ} \mathrm{C}$ & 5,94 & 5,42 \\
\hline
\end{tabular}

El retenido sobre tamiz - pasante malla 230, permite la determinación del porcentaje de arena en el material arcilloso, el cual es indispensable para el correcto secado de los productos. Este ensayo es muy útil porque al conocer la cantidad de arena presente en la muestra, se puede inferir de manera rápida el comportamiento en las diferentes etapas del proceso de producción. A mayores valores de porcentaje de arena es necesario aumentar la temperatura durante la cocción con el fin de disminuir la porosidad, además, a bajas temperaturas de cocción se producirá productos 
con altos valores de absorción de agua y baja resistencia mecánica. Los valores de absorción de agua del 7,51 de la muestra de Villa del Rosario y 7,94 del Zulia, se pueden mejorar aumentando $50^{\circ} \mathrm{C}$ la temperatura de cocción del horno colmena.

En la Tabla 4 se pueden observar que las muestras presentan un porcentaje de arenas similar. De acuerdo con la literatura los porcentajes de arena idóneos para la fabricación de productos cerámicos extruidos oscilan entre el $16 \%$ y un máximo de $35 \%$. Tomando en cuenta el análisis de \% limos, \% arcilla y $\%$ arena se estima que sería necesario agregar arena en un $5 \%$ con una granulometría $(0,6 \mathrm{~mm})$ a las muestras arcillosas de la zona para producir materiales con características dimensionales adecuadas, debido a que su bajo porcentaje de arena genera problemas durante el proceso de secado, extendiendo la duración en el tiempo.

El análisis granulométrico por hidrómetro permite determinar las proporciones de arena, limos y arcilla que contiene el material arcilloso, y de esta manera se puede ubicar en el diagrama de Winkler. La textura del suelo hace referencia a la proporción en función del peso, de las partículas menores a $2 \mathrm{~mm}$ de diámetro, es decir, las arenas, los limos y las arcillas existentes en los horizontes del suelo. Las arenas son aquellas partículas que tiene un diámetro entre $2 \mathrm{~mm}-0,05 \mathrm{~mm}$, los limos $0,05 \mathrm{~mm}$ $-0,002 \mathrm{~mm}$ y las arcillas un diámetro menor a $0,002 \mathrm{~mm}$. Esta información permite clasificar el suelo dentro de un diagrama triangular, según el tamaño de las partículas (Figura 4).

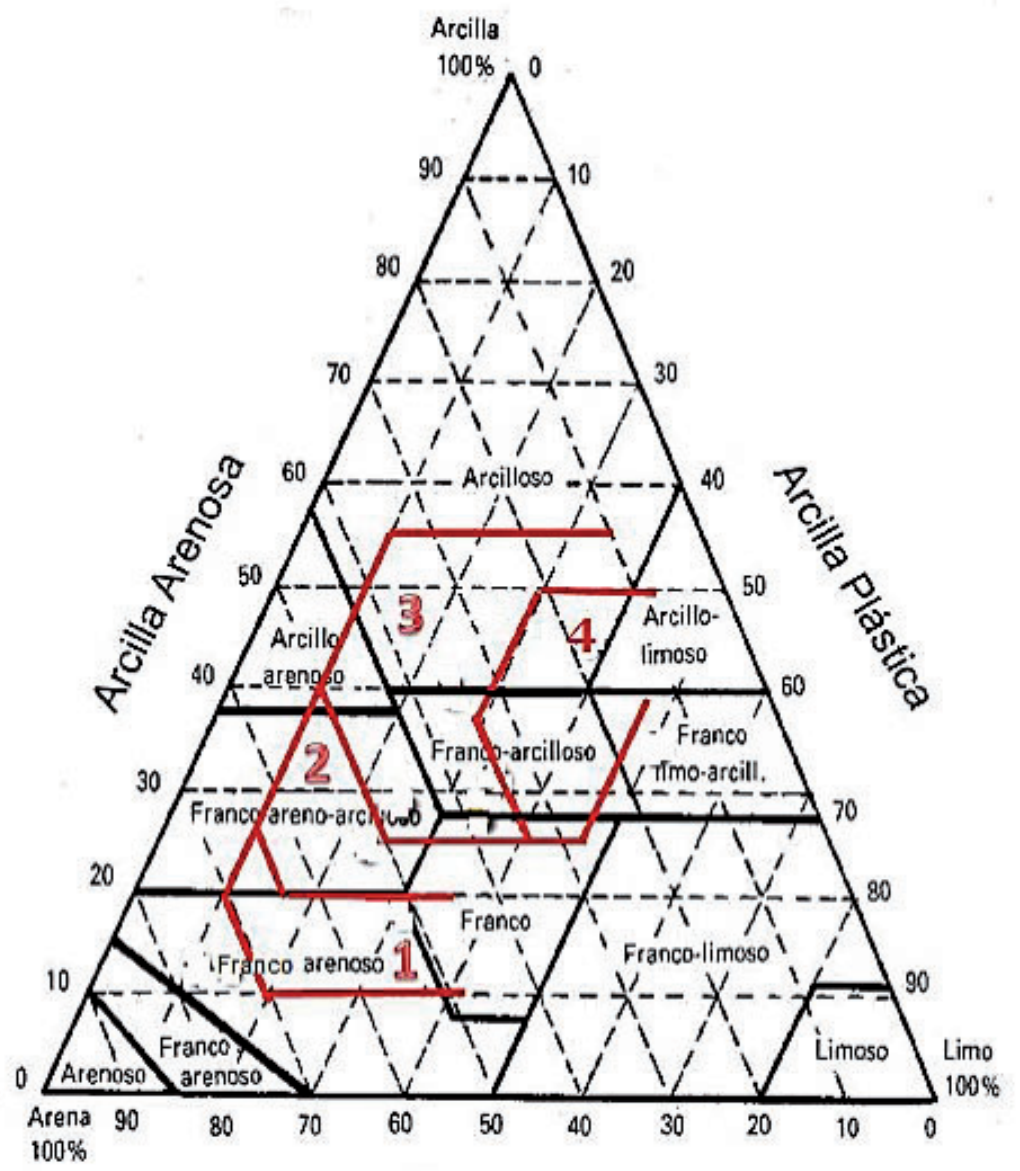

Figura 4. Triángulo textural del suelo mostrando los porcentajes de arcilla, limo y arena de las clases texturales [18]. 
En la Figura 4, se aprecian las zonas de aptitud cerámica de las arcillas, la zona 1 (Z1) ladrillos macizos y ladrillos comunes, zona 2 (Z2) ladrillos de perforación vertical, zona 3 (Z3) tejas, ladrillos de perforación vertical, ladrillos cara vista y baldosas y zona 4 (Z4) ladrillos de perforación horizontal, bovedillas, piezas de paredes delgadas y de gran tamaño. El tamaño de partícula representado en la Tabla 4 permite determinar la aptitud cerámica de las muestras al graficarse el triángulo de Winkler. Se determinó la zona específica Z4, debido a que las muestras presentan una textura clase franco arcilloso la cual se puede utilizar para la fabricación de bloques y piezas de gran formato. Cúcuta y su Área metropolitana se caracterizan por la producción de ladrillos perforados y pisos, al aumentar el \% de arena se ubicaría en Z3, la cual es la zona característica de esos productos.

El índice de plasticidad por el método de Pfefferkorn permite conocer la plasticidad del material arcilloso, este dato es importante para determinar la cantidad de agua que se requiere para la preparación de la pasta. De acuerdo a la Tabla 4 las muestras arcillosas superan el $20 \%$ de plasticidad al aplicar el ensayo, lo que indica que las muestras analizadas presentan capacidad de ser sometidas a esfuerzos para su conformado a través de procesos de extrusión. Los índices de plasticidad de las arcillas de Villa del Rosario y del Zulia dieron como resultado 24,5 usando el método de Pfefferkorn que valida la información de la plasticidad de las arcillas de la formación Guayabo (arcillas del área metropolitana de Cúcuta) [20]. Los valores obtenidos por pérdidas por calcinación son relativamente bajos, estos datos permiten relacionar la presencia de materiales orgánicos que generan gases durante el proceso de cocción, a mayor cantidad de estos, mayor será la presencia de porosidad en las piezas una vez cocidas lo que puede influir en el acabado final de la pieza si la cocción a temperatura máxima no es suficiente para permitir la extracción de los gases en el ciclo de quema. A su vez, estos materiales orgánicos darán mayor capacidad para absorber el agua en el producto terminado, lo que no es deseable. Los resultados están acordes con lo obtenido por FRX. Con respecto a la contracción en seco las arcillas del material presentan elevadas contracciones durante el secado, lo que hace necesario un adecuado control del secadero y la variación en la granulometría con el fin tener un secado controlado. A medida que el material se densifica las contracciones en cocido disminuyen lo que permite obtener productos con bajas absorciones de agua y altos valores en resistencia mecánica.

\section{Conclusiones}

Las muestras arcillosas obtenidas de Villa del Rosario y El Zulia presentan características similares, tales como: las fases cristalinas, los grupos funcionales, el comportamiento térmico y las propiedades cerámicas, a pesar de que se encuentran distantes desde el punto de vista cerámico. Esto conlleva a que se presente un comportamiento cerámico similar, lo que permitiría a las diferentes empresas del sector cerámico del Área Metropolitana de Cúcuta realizar pruebas fisicoquímicas en conjunto, con el fin de producir grandes volúmenes de productos cerámicos para la construcción en común y de esta manera cumplir con las expectativas de producción que se puedan generar en la región.

\section{Referencias Bibliográficas}

[1] Díaz LA, Torrecillas R. Arcillas cerámicas: Una revisión de sus distintos tipos, significados y aplicaciones. Bol. Soc. Esp. Cerám. Vidrio. 2002;41(5):459-70.

[2] Muñoz RA, Muñoz JA, Mancilla $P$, et al. Caracterización fisicoquímica de arcillas del municipio de Guapi-Costa Pacífica Caucana (Colombia). Rev. Acad. Colombi. Cienc. 2007;31(121):537-44.

[3] Servicio Nacional de Aprendizaje SENA. Caracterización Ocupacional Industria de la Arcilla. Mesa Sectorial de Mineria: Fabricación de productos de arcilla para la construcción. 2003.

[4] Gelves JF, Monroy R, Sánchez J, et al. Estudio comparativo de las técnicas de extrusión y prensado como procesos de conformado de productos cerámicos de construcción en el Área Metropolitana de Cúcuta. Bol. Soc. Esp. Cerám. Vidrio. 2013;52(1):48-54.

[5] Díaz JI, Sánchez J, Prato JG. EnergyEnvironmental Diagnosis of the Ceramic Sector Companies in the Metropolitan Area of Cucuta, Norte de Santander, Colombia. Key Engineering Materials. 2016;663:133-9.

[6] Sánchez J, Ramírez P. El cluster de la cerámica del Área Metropolitana de Cúcuta (Primera Ed). Cúcuta-Norte de Santander: Universidad Francisco de Paula Santander; 2013. 
[7] Prato EJ. Planteamiento de modelos productivos para la estandarización de los procesos de producción de las mipymes del sector cerámico del Área Metropolitana de San José de Cúcuta. Cúcuta-Norte de Santander. 2007.

[8] Departamento Nacional de Planeación DNP. Agenda interna para la productividad y la competitividad: Norte de Santander. DNPAgenda Interna. 2007.

[9] Amorós JL, Sánchez E, García-Ten J, et al. Manual para el control de la calidad de materias primas arcillosas. (I. de tecnología cerámica ITC, Ed.) (Segunda). Castellón - España: Instituto de tecnología cerámica ITC; 2004.

[10] Icontec. Norma Técnica Colombiana NTC 4321-3. Ingeniería Civil y Arquitectura. Baldosas Cerámicas. Parte 3. Método de ensayo para determinar la absorción de agua, porosidad aparente, densidad relativa aparente y densidad aparente. Bogotá: Icontec.1998.

[11] Mahmoudi S, Bennour A, Meguebli A, et al. Characterization and traditional ceramic application of clays from the Douiret region in South Tunisia. Applied Clay Science. 2016;127(128):78-87.

[12] Duitama L, Espitia C, Mojica J, Quintero J, et al. Composición mineralógica y química de las arcillas empleadas para Cerámica Roja en las zonas de Medellín, Itagui y Amagá. Rev. Acad. Colomb. 2004;28(109):555-64.

[13] Bernal I, Cabezas H, Espitia C, Mojica J, et al.
Análisis próximo de arcillas para cerámica. Rev. Acad. Colombia. Cienc. 2003;27(105):569-78.

[14] Nayak P, Singh B. Instrumental characterization of clay by XRF, XRD and FTIR. Indian Academy of Sciences. 2007;30(3):235-38.

[15] Saikia B, et al. Fourier transform infrared spectroscopic characterization of kaolinite from Assam and Meghalaya, Northeastem India. J. Mod. Phys. 2010;1:206-10.

[16] Wolff R. Infrared absorption patterns (OH región) of several clay minerals. Mineralogical Notes. The American mineralogist. 1963;50:240-44.

[17] Davarcioglu B. Spectral characterization of non-clay minerals the clays (Central AnatolianTurkey). International Journal of the Physical Sciences. 2011;6(3):511-22.

[18] Muñoz JA, Muñoz RA, Mancilla P, et al. Estudio del procesamiento cerámico de las arcillas de la vereda "La Codicia" (Guapi, Colombia) para potencializar su uso en la elaboración de piezas. Rev. Fac. Ing. Univ. Antioquia. 2007;42:68-78.

[19]Díaz Jl. Informe de resultados Análisis comparativo de las propiedades del conjunto de arcillas del proyecto: Fortalecimiento a 10 empresas del Clúster de la Cerámica de Norte de Santander a través de la estandarización de sus principales productos a la Norma Técnica Colombiana. 2015.

[20] Gelves JF, Sánchez J, Peña G. Comportamiento de las arcillas del área metropolitana de Cúcuta sometidas a proceso de moldeo por extrusión. Respuestas. 2016;14(2):32-8. 